International Journal of Difference Equations (IJDE).

ISSN 0973-6069 Volume 16, Number 1 (2021), pp. 1-10

(C) Research India Publications

https://dx.doi.org/10.37622/IJDE/16.1.2021.1-10

\title{
An Inverse Problems for Nonlinear Evolution Equations: Criteria of Existence of an Invariant Polylinear Controller for a Second-Order Differential System in a Hilbert Space
}

\author{
V.A. Rusanov ${ }^{1}$, A.V. Daneev ${ }^{2}$, A.V. Lakeyev ${ }^{1}$, Yu.É. Linke ${ }^{3}$ \\ ${ }^{1}$ Matrosov Institute for System Dynamics and Control Theory, Siberian Branch of \\ Russian Academy of Sciences, Irkutsk, Russia. \\ ${ }^{2}$ Irkutsk State Transport University, Irkutsk, Russia. \\ ${ }^{3}$ Irkutsk National Research Technical University, Irkutsk, Russia.
}

\begin{abstract}
The solvability of the problem of realizing operator functions of an invariant polylinear controller (IPL-controller) of a non-stationary differential system ( $D$-system) of the second order, which allows for two bundles of dynamic processes of the "trajectory, control" type that are induced in this $D$-system by two different polylinear controllers, to unite these bundles, through the action of the IPL-controller, into a subfamily of admissible solutions of the given $D$ system. The problem under consideration belongs to the type of nonstationary coefficient-operator inverse problems for evolution equations, including hyperbolic ones, in a separable Hilbert space and is solved on the basis of a qualitative study of the properties of continuity and semiadditivity of the Rayleigh-Ritz functional operator. The results obtained have applications in the theory of nonlinear infinite-dimensional adaptive dynamical systems for a class of higher-order polylinear differential models.
\end{abstract}

Keywords: inverse problems of evolution equations, non-stationary secondorder differential system, invariant polylinear controller, the Rayleigh-Ritz functional operator. 


\section{INTRODUCTION}

Inverse problems of evolution equations (IPEE), as a section of the differential realization of dynamical systems, currently represent a fairly extensive area of research [1-10]. In this context, the proposed work continues research [2, 10], while understanding the nature of hyperbolic systems (in the technical sense) helps to clarify and motivate the entire discussion. Its main goal is to study the problem of existence of coefficient operator-functions of an invariant polylinear controller (IPL-controller) of a non-stationary differential system $(D \text {-system })^{1}$ of the second order; however, its results can be extended to stationary $D$-systems [11]. The $I P L$-controller assumes that the controllable $D$-system must contain in the class of its admissible solutions the union of two given bundles of dynamic processes, while each such bundle is unlimited in power (finite/countable/continual) and is induced in this system by its own "individual" polylinear controller.

\section{STATEMENT OF THE PROBLEM OF THE IPL-CONTROLLER}

Further, $\left(X,\|\cdot\|_{X}\right),\left(Y,\|\cdot\|_{Y}\right),\left(Z_{i},\|\cdot\|_{Z}\right), i=1, \ldots, n$ are real separable Hilbert spaces (the pre-Hilbert property are defined by the norms $\left.\|\cdot\|_{X},\|\cdot\|_{Y},\|\cdot\|_{Z}\right), U:=Y \times Z_{1} \times \ldots \times Z_{n}$ is a Hilbert space-product with the norm $\left\|\left(y, z_{1}, \ldots, z_{n}\right)\right\|_{U}:=\left(\|y\|_{Y}^{2}+\sum_{i=1, \ldots, n}\left\|z_{i}\right\|_{Z}^{2}\right)^{1 / 2}, L(Y$, $X)$ is a Banach space with the operator norm $\|\cdot\|_{L(Y, X)}$ of all linear continuous operators acting from $Y$ to $X$ (similarly to $\left(L(X, X),\|\cdot\|_{L(X, X)}\right)$ и $\left.\left(L\left(Z_{i}, X\right),\|\cdot\|_{L(Z, X)}\right)\right), X^{i}$ is the $i$-th Cartesian power of the space $X, \mathrm{~L}\left(X^{i}, Z_{i}\right)$ is the space of all continuous $i$-linear (polylinear) mappings from $X^{i}$ to $Z_{i}$.

Let $T:=\left[t_{0}, t_{1}\right]$ be a segment of the number scale $R$ with Lebesgue measure $\mu$ and $\wp \mu$ be the $\sigma$-algebra of all $\mu$-measurable subsets of $T$. If $(\mathscr{B},\|\cdot\|)$ is some Banach space, then by $\mathrm{L}_{p}(T, \mathscr{B}), p \in[1, \infty)$ we denote the Banach quotient space of $\mu$-equivalence classes of all Bochner integrable mappings $f: T \rightarrow \mathscr{B}$ with the norm $\|f\|_{\mathrm{L}_{p}(T, \bar{B})}:=\left(\int_{T}\right.$ $\left.\|f(\tau)\|^{p} \mu(d \tau)\right)^{1 / p}<\infty$, by $\mathrm{L}_{\infty}(T, \widetilde{\mathcal{B}})$ denotes the space of all (equivalent classes) of $\mu$ measurable and $\mu$-essentially bounded functions from $T$ to $\mathscr{B}$.

Moreover, in what follows $A C^{1}(T, X)$ is the set of all functions $g: T \rightarrow X$ whose first derivative $\dot{g}$ is an absolutely continuous function on $T$ with respect to the measure $\mu$.

Now let us introduce auxiliary constructions related to the notation system. Through

$$
H_{2}:=\mathrm{L}_{2}(T, Y) \times \mathrm{L}_{2}\left(T, Z_{1}\right) \times \ldots \times \mathrm{L}_{2}\left(T, Z_{n}\right)
$$

\footnotetext{
${ }^{1}$ The concepts “ $D$-system”, “ $D$-realization”, “ $D$-model”, “ $D$-equation” are synonyms for us.
} 
we denote the product space with the topology induced by the norm $\left\|\left(h_{0}, \ldots, h_{n}\right)\right\|_{H}:=\left(\int_{T}\left\|\left(h_{0}(\tau), \ldots, h_{n}(\tau)\right)\right\|_{U}^{2} \mu(d \tau)\right)^{1 / 2},\left(h_{0}, \ldots, h_{n}\right) \in H_{2} ;$

it is clear, that $H_{2}$ is a Hilbert space (owing to the construction of the norm $\|\cdot\|_{H}$ ).

We also need a Banach product space

$\mathbf{L}_{2}:=\mathrm{L}_{2}(T, L(Y, X)) \times \mathrm{L}_{2}\left(T, L\left(Z_{1}, X\right)\right) \times \ldots \times \mathrm{L}_{2}\left(T, L\left(Z_{n}, X\right)\right)$

of $\mu$-equivalence classes of ordered systems of operator functions with the norm:

$\left\|\left(B_{0}, \ldots, B_{n}\right)\right\|_{\mathbf{L}}:=\left(\int_{T}\left(\left\|B_{0}(\tau)\right\|_{L(Y, X)}^{2}+\sum_{i=1, \ldots, n}\left\|B_{i}(\tau)\right\|_{L(Z, X)}^{2}\right) \mu(d \tau)\right)^{1 / 2}$.

Now let us accept that the operator functions

$A_{0}, A_{1} \in \mathrm{L}_{1}(T, L(X, X)), A_{2} \in \mathrm{L}_{\infty}(T, L(X, X))$,

$\mu\left\{t \in T: A_{2}(t)=0 \in L(X, X)\right\}=0$

are given along with the related linear operator $D: A C^{1}(T, X) \rightarrow \mathrm{L}_{1}(T, X)$ of the form:

$x \mapsto \boldsymbol{D}(g):=A_{2} \ddot{g}+A_{1} \dot{g}+A_{0} g$.

Further, we assume that by fixing $\left(B_{01}, \ldots, B_{n 1}\right),\left(B_{02}, \ldots, B_{n 2}\right) \in \mathbf{L}_{2}, \mathrm{D}_{i} \in \mathrm{L}\left(X^{i}, Z_{i}\right), i$ $=1, \ldots, n$, we define polylinear controllers $\boldsymbol{P} \boldsymbol{L}_{j}: A C^{1}(T, X) \times \mathrm{L}_{2}(T, Y) \rightarrow \mathrm{L}_{1}(T, X), j=$ 1, 2 of the form:

$(g, u) \mapsto \boldsymbol{P} \boldsymbol{L}_{j}(g, u): B_{0 j} u+\sum_{i=1, \ldots, n} B_{i j} \mathrm{D}_{i}(g, \ldots, g), j=1,2$,

$\left(B_{01}, \ldots, B_{n 1}\right) \neq\left(B_{02}, \ldots, B_{n 2}\right)$.

Moreover, we accept (all other things being equal) a "behavioristic" agreement that

$N_{1} \subset\left\{\left(x, u, \mathrm{D}_{1}(x), \ldots, \mathrm{D}_{n}(x, \ldots, x)\right) \in A C^{1}(T, X) \times H_{2}\right\}, C \operatorname{Card} N_{1} \leq \exp \aleph_{0}$,

$N_{2} \subset\left\{\left(x, u, \mathbb{D}_{1}(x), \ldots, \mathbb{D}_{n}(x, \ldots, x)\right) \in A C^{1}(T, X) \times H_{2}\right\}, C \operatorname{Card} N_{2} \leq \exp \aleph_{0}$,

$N_{1} \cap N_{2}=\varnothing$,

are some given dynamic bundles (sometimes, roughly speaking, we will say "solution sets"), induced from two $D$-systems having the representation:

$\boldsymbol{D}(x)=\boldsymbol{P} \boldsymbol{L}_{1}(x, u),\left(x, u, \mathrm{D}_{1}(x), \ldots, \mathrm{D}_{n}(x, \ldots, x)\right) \in N_{1}$, 
$\boldsymbol{D}(x)=\boldsymbol{P} \boldsymbol{L}_{2}(x, u),\left(x, u, \mathrm{D}_{1}(x), \ldots, \mathrm{D}_{n}(x, \ldots, x)\right) \in N_{2} ;$

here, as well as below in $D$-equation (2), equalities are considered as identities in $\mathrm{L}_{1}(T, X)$.

Let us further agree to distinguish in the notation the equivalence class $(\bmod \mu)$

$\left(x, u, \mathrm{D}_{1}(x), \ldots, \mathrm{D}_{n}(x, \ldots, x)\right) \in A C^{1}(T, X) \times H_{2}$

from a specific representative of this class, namely, the "individual" vector function

$t \mapsto\left(x(t), u(t), \mathrm{D}_{1}(x(t)), \ldots, \mathrm{D}_{n}(x(t), \ldots, x(t))\right)$.

Consider the problem: define in terms of the bundle $\tilde{N}:=N_{1} \cup N_{2}$ the conditions for the existence of a tuple $\left(\widetilde{B}_{0}, \ldots, \widetilde{B}_{n}\right) \in \mathbf{L}_{2}$ (operator coefficients of the IPLcontroller), for which the following takes place:

$\boldsymbol{D}(x)=\boldsymbol{I P L}(x, u):=\widetilde{B}_{0} u+\sum_{i=1, \ldots, n} \tilde{B}_{i} \mathrm{D}_{i}(x, \ldots, x),\left(x, u, \mathrm{D}_{1}(x), \ldots, \mathrm{D}_{n}(x, \ldots, x)\right) \in \tilde{N}$

The solution of the inverse Problem (2) leads to theoretical schemes (see Theorems 1, 2 below), explaining the physical nature of adaptive control systems, simultaneously developing mathematical intuition in IPEE for modeling nonlinear hyperbolic systems $[1,12]$, including number, for models with $I P L$-controllers possessing the minimal operator norm $\mathrm{L}\left(X^{i} \times Y, Z_{i}\right)[13]$.

Remark 1. Note that there are no structural obstacles to the extension of the results obtained below to $I P L_{-}$-controllers that include polylinear operators from $\mathrm{L}\left(X^{i} \times Y, Z_{i}\right)$ and contain $k$-times $(k \leq i)$ derivative $d x / d t$ and 1-time programmed control $u$ as an additional variable; it is clear that in this formulation, $\mathrm{D}(x, \ldots, d x / d t, \ldots, u) \in \mathrm{L}_{2}(T$, $\left.Z_{i}\right)$ for any $\mathrm{D} \in \mathrm{L}\left(X^{i} \times Y, Z_{i}\right)$. Moreover, if within the framework of IPEE we pose the problem of solvability of the realization of polylinear operators themselves from $\mathrm{L}(X$ $\left.{ }^{i} \times Y, Z_{i}\right), i=1, \ldots, n$, then the basis of the mathematical apparatus can be the tensor product of separable Hilbert spaces [9], since its structure reduces the study of polylinear mappings to the study of linear mappings by introducing the operation of $M_{2}$-extendability [6] on the category of special linear spaces.

\section{AROUND THE ANALYTICAL CONDITIONS OF THE EXISTENCE OF THE IPL-CONTROLLER}

We denote by $\mathrm{L}(T, R)$ the space of $\mu$-equivalence classes of all real $\mu$-measurable functions on $T$ and let $\leq_{\mathrm{L}}$ be a quasi-ordering in $\mathrm{L}(T, R)$ such that $\phi_{1} \leq_{\mathrm{L}} \phi_{2}$ if $\phi_{1}(t) \leq$ 
$\phi_{2}(t) \mu$-almost everywhere in $T$. The smallest bound for the subset $W \subset \mathrm{L}(T, R)$ is denoted by $\sup _{L} W$ if this bound exists for the subset $W$ in the structure of the partial ordering $\leq_{\mathrm{L}}$.

Definition 1 [2, 8]. Consider on $\Pi:=A C^{1}(T, X) \times H_{2}$ a nonlinear operator $\Psi: \Pi \rightarrow \mathrm{L}(T, R)$, constructed according to the following rule:

$$
t \mapsto \Psi(g, h)(t):=\left\{\begin{array}{l}
\|D(g)(t)\|_{X} \times\|h(t)\|_{U}^{-1} \text { if } h(t) \neq 0 \in U ; \\
0 \in R, \text { if } h(t)=0 \in U,
\end{array}\right.
$$

where $g \in A C^{1}(T, X), h \in H_{2}$. Following [8], operator (3) will be called the Rayleigh-Ritz operator.

From the functional construction (3) it follows that the Rayleigh-Ritz operator satisfies the simple (but important $[8,9]$ ) relations:

$\chi \varnothing \leq_{\mathrm{L}} \Psi(\phi), \Psi(r \phi)=\Psi(\phi), \phi \in \Pi, 0 \neq r \in R$,

where $\chi \varnothing \in \mathrm{L}(T, R)$ is the characteristic function of the empty set $\varnothing \in \wp \mu$.

Now, before going any further, let us introduce some additional terminology.

Definition 2. A Rayleigh-Ritz operator is said to be semiadditive with weight $p \in R$ on the set $E \subset \Pi$, if for any pair $\left(\phi^{\prime}, \phi^{\prime \prime}\right) \in E \times E$ the following $\leq_{\mathrm{L}}$-inequality holds:

$\Psi\left(\phi^{\prime}+\phi^{\prime \prime}\right) \leq_{\mathrm{L}} p \Psi\left(\phi^{\prime}\right)+p \Psi\left(\phi^{\prime \prime}\right)$.

Lemma 1. ${ }^{2}$ Semiadditivity with a fixed weight of the Rayleigh-Ritz operator is a finite property for subsets of $\Pi$.

Remark 2. The relationship between Lemma 1 and the classical Teichmüller-Tukey ${ }^{3}$ Lemma [14, p. 28] leads to an important geometric characteristic of the semiadditivity of the Rayleigh-Ritz operator, namely: in $\Pi$ there are maximal sets on which operator (3) is semiadditive with some weight $p>0$, and these sets cannot be linear in the case of $p \in(0,1)$; to make sure, it suffices to consider (when $E \subset \Pi$ a linear set, that is, Span $E=E$ ) the action of $\Psi$ on the pair $(\phi, \mathbf{0}) \in E \times E, \phi \neq \mathbf{0}$ (here and below, $\mathbf{0}$ is a zero vector from $\Pi$ ) except for the trivial variant $E=\{\boldsymbol{0}\} \subset \Pi$. That is why in Lemma 2 below (and by default, further) it is assumed that the semiadditivity weight of the operator $\Psi$ is some fixed number $p \in[1, \infty)$.

\footnotetext{
${ }^{2}$ Recall that property $\mathscr{P}$ is a property of finite nature in $\Pi$ if the empty set has this property, and $E \subset \Pi$ has property $\mathscr{P}$ if and only if every finite subset of $E$ has it.

${ }^{3}$ The Teichmüller-Tukey Lemma is an alternative form of the Axiom of Choice [14, p. 28].
} 
Lemma 2. Let $\phi \in \Pi, \phi \neq \mathbf{0} u p \in[1, \infty)$. Then there exists a linear set $E \subset \Pi$ that is maximal with respect to a set-theoretic inclusion and contains a function $\phi$, and the Rayleigh-Ritz functional operator will be semiadditive on $E$ with weight $p$.

Example. Let us construct a triple $(\Psi, \bar{N}, p)$, for which operator (3) is semiadditive with weight $p=1$ on an infinite-dimensional linear manifold $\bar{N} \subset \Pi$, closed in $\left(\mathrm{L}_{2}(T\right.$, $\left.X),\|\cdot\|_{\mathrm{L}_{2}(T, X)}\right) \times\left(H_{2},\|\cdot\|_{H}\right)$ with the same product topology. Let $\chi_{T}$ be the characteristic function of the interval $T$, and let the operator functions $A_{0}, A_{1}, A_{2}$ in the operator $\boldsymbol{D}(\cdot)$ have the representations: $A_{0}=A_{1}=0 \in \mathrm{L}_{1}(T, L(X, X)), A_{2}=\Gamma \in L(X, X)$, where the operator $\Gamma$ implements a nonzero homothety on $X$. Further, let $\bar{N}$ be the linear dynamical bundle (Span $\bar{N}=\bar{N}$ ) induced by all solutions of the $D$-system (2) with the operators $A_{0}, A_{1}, A_{2}$ and $B_{1}=\mathrm{id}_{X}, \mathrm{D}_{1}=\Gamma, \mathrm{D}_{i}=0 \in \mathbb{L}\left(X^{i}, Z_{i}\right), i=2, \ldots, n, u(\cdot)=0$ $\in \mathrm{L}_{2}(T, Y)$. In this formulation, the operator $\Psi$ is semi-additive on $\bar{N}$ with weight $p=$ 1 , since $\Psi(\phi)=\chi_{T}$ for any vector function $\phi \in \bar{N}$.

Now let us consider the continuity property of the Rayleigh-Ritz operator [8]. For this, on the linear space $\mathrm{L}(T, R)$, consider the vector topology generated by the convergence in measure $\mu$. It is well known (see [8]) that this topology is generated by a quasinorm of the form:

$\rho\left(f_{1}, f_{2}\right):=\int_{T}\left(1+\left|f_{1}(t)-f_{2}(t)\right|\right)^{-1}\left|f_{1}(t)-f_{2}(t)\right| \mu(d t)$

in this context $(\mathrm{L}(T, R), \rho)$ is a complete quasi-normed space.

In what follows, for any function $f \in \mathrm{L}(T, R)$, we denote supp $f:=\{x \in X: f(x) \neq 0\}$ its support defined up to a set of measure zero.

Lemma 3. Let $\left(\Pi, \rho^{*}\right)$ be a metric space with metric $\rho^{*}: \Pi \times \Pi \rightarrow R$ of the form:

$\rho^{*}((g, h),(\hat{g}, \hat{h})):=\rho\left(\|g\|_{X},\|\hat{g}\|_{X}\right)+\rho\left(\|h\|_{U},\|\hat{h}\|_{U}\right)+$

$+\mu\left(\operatorname{supp}\|h\|_{U} \Delta \operatorname{supp}\|\hat{h}\|_{U}\right), g, \hat{g} \in A C^{1}(T, X), h, \hat{h} \in H_{2}$,

where (supp $\|h\|_{U} \Delta$ supp $\left.\|\hat{h}\|_{U}\right) \in \wp_{\mu}$ is the symmetric difference of the supports supp $\|h\|_{U}$ and $\operatorname{supp}\|\hat{h}\|_{U}$.

Then the following statements are true:

(i) the metric $\rho^{*}$ is not a quasinorm, and the topology generated by $\rho^{*}$ will not be vector (the operations of the vector space $\Pi$ are not continuous in this topology);

(ii) the Rayleigh-Ritz operator $\Psi:\left(\Pi, \rho^{*}\right) \rightarrow(\mathrm{L}(T, R), \rho)$ is continuous. 
Corollary 1. Let $\Pi^{*}$ be a finite-dimensional linear manifold in $\Pi$. Then:

(i) the metric space $\left(\Pi^{*}, \rho^{*}\right)$ is incomplete, and the fundamental sequences from $\left(\Pi^{*}, \rho^{*}\right)$ are contained in the class of Cauchy's sequences of the space $\left(\Pi^{*}, \mathcal{T}\right)$, where $T$ is the topology induced in $\Pi^{*}$ from the space $\left(\mathrm{L}_{2}(T, X),\|\cdot\|_{\mathrm{L}_{2}(T, X)}\right) \times\left(H_{2},\|\cdot\|_{H}\right)$;

(ii) the metric $\rho^{*}$ is a quasinorm, and the topology $T *$ generated by $\rho^{*}$, is vector if $\forall f \in \Pi^{*} \backslash\{\boldsymbol{0}\}: \operatorname{supp}\|f\|_{U}:=T(\bmod \mu)$,

moreover, $\mathcal{T}^{*}=\mathcal{T}$, and the image of the Rayleigh-Ritz operator $\Psi\left[\Pi^{*}\right]$ is a $\rho$ quasinormal compactum.

Further, in the construction of an $i^{-1}$-dense $(i=1,2, \ldots)$ subset in $\left(\Pi^{*}, \rho^{*}\right)$ we follow [14, p. 395].

A necessary condition for the existence of an IPL-controller:

Theorem 1. If Card $N_{j}<\aleph_{0}, j=1,2$ and supp $\|f\|_{U}:=T(\bmod \mu), f \in \operatorname{Span} \tilde{N} \backslash\{\boldsymbol{0}\}$, then the following statement is true:

inverse Problem (2) is solvable, i.e. $\exists\left(\tilde{B}_{0}, \ldots, \widetilde{B}_{n}\right) \in \mathbf{L}_{2} \Rightarrow$

$\Rightarrow \exists \sup _{\mathrm{L}} \Psi[\operatorname{Span} \tilde{N}] \Leftrightarrow \rho\left(\sup _{\mathrm{L}} W_{n}, \sup _{\mathrm{L}} W_{m}\right) \underset{n, m \rightarrow \infty}{\longrightarrow} 0$,

where $W_{n}=\bigcup\left\{V_{i}: i=1, \ldots, n\right\}, V_{i}(i=1,2, \ldots)$ is a finite $i^{-1}$-dense subset of $(\Psi[$ Span $\left.\tilde{N}], \rho^{*}\right)$.

It is easy to show that the bound $\sup _{\mathrm{L}} W_{n}$ for $W_{n}=\left\{w_{(1)}, \ldots, w_{(m)}\right\} \subset \mathrm{L}(T, R)$ is equal to the function $w^{(m)}$, which can be calculated using the following recursive rule:

$w^{(1)}:=w_{(1)}, w^{(j+1)}:=2^{-1}\left(w^{(j)}+w_{(j+1)}+\left|w^{(j)}-w_{(j+1)}\right|\right), j=2, \ldots, m-1$.

A sufficient condition for the existence of an IPL-controller:

Theorem 2. D-model (2) exists if the Rayleigh-Ritz operator is semiadditive with some weight on the linear span Span $\tilde{N}$.

Corollary 2. Let $N_{1}, \ldots, N_{k} \subset \Pi$, Card $N_{j} \leq \exp \aleph_{0}, j=1, \ldots, k$ and for each index $j$ the set of dynamic processes $N_{j}(j=1, \ldots, k)$ has a $D$-realization of the form:

$\exists\left(B_{0 j}, \ldots, B_{n j}\right) \in \mathbf{L}_{2} \forall\left(x, u, \mathbb{D}_{1}(x), \ldots, \mathbb{D}_{n}(x, \ldots, x)\right) \in N_{j}:$

$\boldsymbol{D}(x)=\boldsymbol{P} \boldsymbol{L}_{j}(x, u)=B_{0 j} u+\sum_{i=1, \ldots, n} B_{i j} \mathrm{D}_{i}(x, \ldots, x)$, 
$\left(B_{0 j}, \ldots, B_{n j}\right) \neq\left(B_{0 l}, \ldots, B_{n l}\right), j \neq l(j, l \in\{1, \ldots, k\})$.

Then: $\bar{N}:=\bigcup N_{j}$ is a family of solutions of the D-equation (2) for some tuple $\left(\tilde{B}_{0}, \ldots, \widetilde{B}_{n}\right) \in \mathbf{L}_{2}$, if the operator $\Psi$ is semiadditive with some weight on Span $\bar{N}$.

\section{CONCLUSION}

The current period of IPEE development in a separable Hilbert space is largely associated with the creation of a new language - the theory of extensions of nonstationary $M_{p}$-operators ${ }^{4}$ in an infinite-dimensional setting [15]. This theory substantially restructured and strengthened the system and theoretical foundations of IPEE methods in Hilbert space and provided a connection between the geometric ideas of the extendability of $M_{p}$-operators and the qualitative theory of nonlinear differential equations in infinite-dimensional spaces, with an emphasis on applications [16] rather than on achieving the maximum mathematical generality of presentation of this area of inverse problems of modern mathematical physics [12]. In general, it would not be an exaggeration to say that the "polylinear structures" of this geometric theory, which have been promoted above, provide at least aesthetic satisfaction, since the analyst pursuing (among other things) purely aesthetic goals, as a rule, contributes to the creation a new formal language, more adapted to maximally satisfy the mathematical needs of a physicist.

In this context, Corollary 2 allows us to construct an algebra of sets [17] of dynamical processes with unit $\bigcup N_{j}$, all elements of which have a $D$-realization (2). In this case, the question of the "individual" characteristic feature of $D$-realization (4) for each individual sheaf $N_{j}(j=1, \ldots, k)$ is especially simply (constructively) solved for oneelement bundles $N_{j}=\left\{\left(x, u, \mathrm{D}_{1}(x), \ldots, \mathrm{D}_{n}(x, \ldots, x)\right)_{j}\right\}$ by checking (see Theorem 2 [2]) the conditions:

$\Psi\left(\left(x, u, \mathrm{D}_{1}(x), \ldots, \mathrm{D}_{n}(x, \ldots, x)\right)_{j}\right) \in \mathrm{L}_{2}(T, R), j=1, \ldots, k$.

If these relations (or some of them) are not satisfied, then we can pose the problem of finding $i$-linear operators $\mathrm{D}_{i}^{\#} \in \mathrm{L}\left(X^{i}, Z_{i}\right), i=1, \ldots, n$, functions $f_{i l}: X \rightarrow X(i, l$ $\in\{1, \ldots, n\})$, as well as a new "source of influence" $t \mapsto u^{\#}(t)$, which provide the above conditions, i.e.

$\Psi\left(\left(x, u^{\#}, \mathrm{D}_{1}^{\#}\left(f_{11}\left(y_{1}\right)\right), \ldots, \mathrm{D}_{n}^{\#}\left(f_{n 1}\left(y_{n}\right), \ldots, f_{n n}\left(y_{n}\right)\right)\right)_{j}\right) \in \mathrm{L}_{2}(T, R), j=1, \ldots, k$.

${ }^{4}$ The geometric ideas of this theory for finite-dimensional spaces were proposed by A.V. Lakeev in [21]. 
Methodologically, this problem can be interpreted as an adaptive adjustment of the structure of the polylinear components of equation (2), which is attractive in $D$ modeling of nonlinear neurodynamic processes [18, 19]; in this context, see Examples $1-4[20]$.

Acknowledgements: This work was supported by the Russian Foundation for Basic Research (Project No. 19-01-00301).

\section{REFERENCES}

[1] Yu.E. Anikonov and M.V. Neshchadim. On analytical methods in the theory of inverse problems for hyperbolic equations. I, II. Journal of Applied and Industrial Mathematics. 2011. Vol. 5. No. 4. P. 506-518; J. Appl. Indust. Math. 2012. Vol. 6. No. 1. P. 6-11.

[2] A.V. Lakeev, Yu.É. Linke and V.A. Rusanov. Realization of a polylinear controller as a second-order differential system in a Hilbert space. Differential Equations. 2017. Vol. 53. No. 8. P. 1070-1081.

[3] V.A. Rusanov, A.V. Daneev and Yu.E. Linke. To the geometrical theory of the differential realization of dynamic processes in a Hilbert space. Cybernetics and Systems Analysis. 2017. Vol. 53. No. 4. P. 554-564.

[4] A.T. Ramazanova, H.F. Kuliyev and A. Roesch. An inverse problem for determining right hand side of equations for hyperbolic equation of fourth order. Advances in Differential Equations and Control Processes. 2019. Vol. 20. No. 2. P. $143-16$

[5] V.A. Rusanov, A.V. Daneev and Yu.É. Linke. Adjustment optimization for a model of differential realization of a multidimensional second-order system. Differential Equations. 2019. Vol. 55. No. 10. P. 1390-1396.

[6] V.A. Rusanov, A.V. Banshchikov, A.V. Daneev and A.V. Lakeyev. Maximum entropy principle in the differential second-order realization of a nonstationary bilinear system. Advances in Differential Equations and Control Processes. 2019. Vol. 20. No. 2. P. 223-248.

[7] A.V. Lakeev, Yu.E. Linke and V.A. Rusanov. On the differential realization of a second-order bilinear system in a Hilbert space. Journal of Applied and Industrial Mathematics. 2019. Vol. 13. No. 2. P. 261-269.

[8] V.A. Rusanov, A.V. Daneev, A.V. Lakeyev and Yu.É. Linke. On the theory of differential realization: Criteria of continuity of the nonlinear Rayleigh-Ritz operator. International Journal of Functional Analysis, Operator Theory and Applications. 2020. Vol. 12. No. 1. P. 1-22.

[9] A.V. Daneev, A.V. Lakeyev and V.A. Rusanov. Existence of a bilinear differential realization in the constructions of tensor product of Hilbert spaces. WSEAS Transactions on Mathematics. 2020. Vol. 19. P. 99-107. 
[10] V.A. Rusanov, A.V. Daneev, A.V. Lakeyev and Yu.É. Linke. To existence of a nonstationary quasi-linear vector field realizing the expansion of a control trajectory bundle in Hilbert space. WSEAS Transactions on Systems. 2020. Vol. 19. P. 115-120.

[11] V.A. Rusanov, A.V. Daneev, A.V. Lakeyev and V.N. Sizykh. Higher-order differential realization of polylinear-controlled dynamic processes in a Hilbert space. Advances in Differential Equations and Control Processes. 2018. Vol. 19. No. 3. P. 263-274.

[12] S.I. Kabanikhin. Inverse and Ill-Posed Problems. Novosibirsk: Siberian Scientific Publishing Department, 2009 (in Russian).

[13] V.A. Rusanov, L.V. Antonova, A.V. Daneev and A.S. Mironov. Differential realization with a minimum operator norm of a controlled dynamic process. Advances in Differential Equations and Control Processes. 2013. Vol. 11. No. 1. P. $1-40$.

[14] R. Engelking. General Topology. Warszawa: PWH, 1985; Moscow: Mir, 1986 (in Russian).

[15] V.A. Rusanov, A.V. Lakeev and Yu.É. Linke. Existence of a differential realization of a dynamical system in a Banach space in the constructions of extensions to $M_{p}$-operators. Differential Equations. 2013. Vol. 49. No. 3. P. 346-358.

[16] A.V. Daneev, A.V. Lakeyev and V.A. Rusanov. On the differential simulation of the second-order bilinear system: a tensor approach. Proceedings the 2nd International Conference on Mathematics and Computers in Science and Engineering (MACISE). Madrid, Spain. IEEE Catalog Number: CFP20S31ART. 2020. P. 318-321.

[17] V.A. Rusanov. Algebra of sets of dynamic processes with differential realizations in a Hilbert space. Doklady Mathematics. 2010. Vol. 82. No. 1. P. 676-677.

[18] S. Brzychczy and R. Poznanski. Mathematical Neuroscience. Academic Press. 2013.

[19] G. Valle. An integrated brain-machine interface platform with thousands of channels. J. Med. Internet Res. 2019. Vol. 21. No. 10: e16194. (doi: $10.2196 / 16194)$

[20] V.A. Rusanov, A.V. Daneev, Yu.É. Linke and P.A. Plesnyov. Existence of a bilinear delay differential realization of nonlinear neurodynamic process in the constructions of entropy Rayleigh-Ritz operator. Advances in Dynamical Systems and Applications. 2020. Vol. 15. No. 2. P. 199-215.

[21] A.V. Daneev, A.V. Lakeev and V.A. Rusanov. On the theory of realization of strong differential models. II. Journal of Applied and Industrial Mathematics. 2007. Vol. 1. No. 3. P. 283-292. 